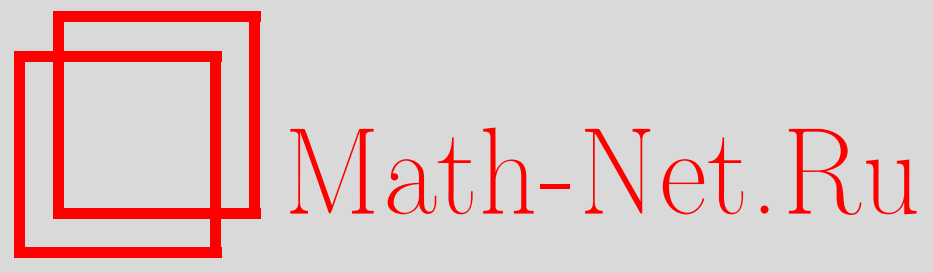

И. Ю. Миронюк, Л. А. Усов, Инвариант линии торможения при стационарном обтекании тела завихренным потоком идеальной несжимаемой жидкости, Вестн. Сам. гос. техн. ун-та. Сер. Физ.мат. науки, 2020, номер 4, 780-789

DOI: https://doi.org/10.14498/vsgtu1815

Использование Общероссийского математического портала MathNet.Ru подразумевает, что вы прочитали и согласны с пользовательским соглашением

http: //www . mathnet.ru/rus/agreement

Параметры загрузки:

IP : 18.234 .156 .22

26 апреля 2023 г., 13:51:38

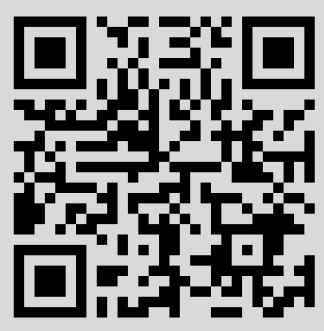


УДК 532.5.011

\title{
Инвариант линии торможения при стационарном обтекании тела завихренным потоком идеальной несжимаемой жидкости
}

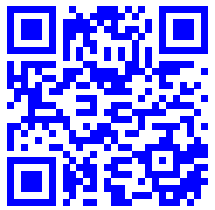

\author{
(С) И. Ю. Миронюк, Л. А. Усов
}

Московский физико-технический институт (национальный исследовательский университет),

Россия, 141701, Московская обл., Долгопрудный, Институтский пер., 9.

\section{Аннотация}

С использованием уравнений Эйлера исследуется линия торможения (критическая линия) в общем пространственном случае стационарного обтекания тела с гладкой выпуклой носовой частью несжимаемой жидкостью. Предполагается, что в некоторой окрестности точки торможения (критической точки) всюду, за исключением точки торможения, скорость жидкости отлична от нуля, и что все линии тока на поверхности тела в этой окрестности начинаются в точке торможения.

Доказываются следующие три утверждения. 1) Если на некотором отрезке вихревой линии завихренность не обращается в нуль, то величина скорости жидкости на этом отрезке либо тождественно равна нулю, либо отлична от нуля во всех точках отрезка вихревой линии (скоростная альтернатива). 2) Завихренность в точке торможения равна нулю. 3) На линии торможения завихренность коллинеарна скорости и отношение величины завихренности к величине скорости одинаково во всех точках линии торможения (инвариант линии торможения).

На основании полученных результатов делается вывод о невозможности стационарного обтекания тела вихревым потоком, в котором скорость и завихренность не коллинеарны. Однако вопрос о завихренности в точке торможения в плоскопараллельных течениях остается открытым, поскольку принятое предположение об отличии от нуля скорости жидкости в некоторой окрестности точки торможения всюду, кроме самой точки торможения, исключает из рассмотрения плоскопараллельные течения.

Ключевые слова: уравнения Эйлера, теоремы Гельмгольца о вихрях, критерий Зоравского, критическая линия тока, линия торможения.

Получение: 30 июля 2020 г. / Исправление: 15 августа 2020 г. Принятие: 16 ноября 2020 г. / Публикация онлайн: 25 декабря 2020 г.

\section{Краткое сообщение}

( () (7) Контент публикуется на условиях лицензии Creative Commons Attribution 4.0 International (https://creativecommons.org/licenses/by/4.0/deed.ru)

\section{Образец для цитирования}

Миронюк И. Ю., Усов Л. А. Инвариант линии торможения при стационарном обтекании тела завихренным потоком идеальной несжимаемой жидкости // Becmн. Сам. гос. техн. ун-та. Сер. Физ.-мат. науки, 2020. Т. 24, № 4. С. 780-789. https://doi.org/10.14498/vsgtu1815.

\section{Сведения об авторах}

Игорь Юрьевич Миронюк (10) https://orcid.org/0000-0002-3835-6059

студент; факультет аэромеханики и летательной техники; e-mail:mironyuck.ig@yandex.ru

Лев Александрович Усов (10) https://orcid.org/0000-0001-7316-7328

студент; факультет аэромеханики и летательной техники; e-mail: usov.la@phystech.edu 
Введение. Современное состояние вычислительной техники и уровень развития численных методов позволяют исследовать течения жидкости и газа в рамках моделей, гораздо более сложных, чем модель идеальной несжимаемой жидкости. Однако некоторые процессы и в настоящее время с успехом изучаются в рамках этой модели [1-7]. Наряду с поиском точных решений уравнений движения вязкого газа $[8,2]$ исследователи ищут точные решения уравнений движения идеальной несжимаемой жидкости $[1,3]$. Все это говорит о том, что исследование общих свойств течений несжимаемой жидкости в рамках уравнений Эйлера до сих пор остается актуальным. Данная статья посвящена свойствам завихренности на линии торможения при наличии завихренности в потоке, обтекающем несимметричное тело или осесимметричное тело под углом атаки. В отличие от осесимметричного обтекания, где линия торможения лежит на оси симметрии (рис. а), в рассматриваемом случае линия торможения искривлена (рис. b), и в течении отсутствует симметрия, что значительно усложняет исследование.

Недавно в статье [12] в общем 3D-случае обнаружены некоторые замечательные свойства завихренности на линии торможения в изоэнергетических течениях идеального газа. Результаты [12] нельзя непосредственно применить к идеальной несжимаемой жидкости. Однако авторы данной статьи заметили, что два шага доказательства [12] при надлежащем видоизменении можно применить и к идеальной несжимаемой жидкости. Один из этих шагов «повторен» ниже в третьем разделе. Что касается другого шага доказательства [12], то речь идет о доказательстве равенства нулю завихренности в точке торможения. Для идеальной несжимаемой жидкости можно было бы применить видоизмененное доказательство [12] этого факта. Однако в данной статье предложено совершенно другое доказательство, основанное на неизвестном ранее свойстве (названном ниже скоростной альтернативой).

Наряду с использованием перечисленных выше идей [12] данная статья содержит новые оригинальные доказательства, и обнаруженные в ней свойства (скоростная альтернатива, инвариант линии торможения и вывод о невозможности стационарных обтеканий тел некоторыми вихревыми потоками) ранее были неизвестны, не являются видоизмененными результатами какихлибо других работ и получены впервые.

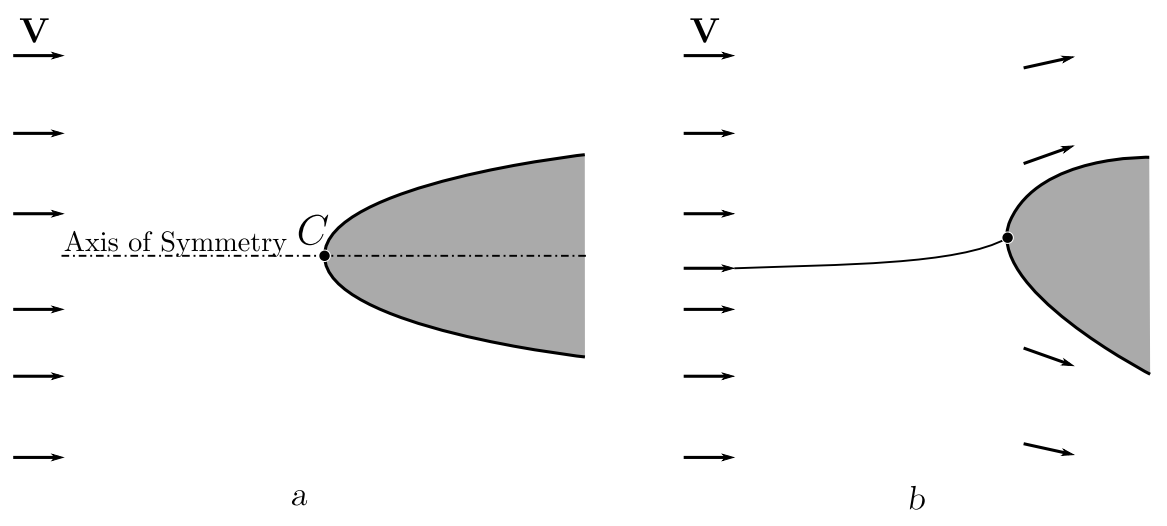

Носовая часть тела в потоке: а) осесимметричное обтекание; b) неосесимметричное обтекание [The nasal part of the body in the stream: a) axisymmetric flow; b) non-axisymmetric flow] 
1. Уравнения движения. Рассмотрим обтекание тела с гладкой выпуклой носовой частью вихревым потоком идеальной несжимаемой жидкости. Уравнения Эйлера стационарного движения идеальной несжимаемой жидкости запишем в форме Громеки-Ламба [13]:

$$
\boldsymbol{\omega} \times \mathbf{V}=-\nabla B
$$

где $\mathbf{V}$ - скорость, $\boldsymbol{\omega}=\operatorname{rot} \mathbf{V}$ - завихренность, $B=p \rho^{-1}+\frac{1}{2} \mathbf{V}^{2}-$ функция Бернулли, где, в свою очередь, $p$ - давление, $\rho=$ const - плотность. Дополним (1) уравнением неразрывности

$$
\operatorname{div} \mathbf{V}=0
$$

Гидродинамические функции $\mathbf{V}$ и $p$ предполагаются дважды непрерывно дифференцируемыми по пространственным координатам в замыкании ${ }^{1}$ некоторой области течения $G$, содержащей линию торможения. Под производными гидродинамических функций на поверхности тела понимаются их непрерывные продолжения из $G$. Замыкание области $G$ обозначим $\bar{G}$.

2. Скоростная альтернатива. Применение операции ротора к обеим частям равенства (1) с учетом (2) и тождества $\operatorname{div} \boldsymbol{\omega}=0$ дает

$$
(\mathbf{V} \cdot \boldsymbol{\nabla}) \boldsymbol{\omega}-(\boldsymbol{\omega} \cdot \boldsymbol{\nabla}) \mathbf{V}=0
$$

Представим завихренность в виде $\boldsymbol{\omega}=\omega \mathbf{e},|\mathbf{e}|=1$. Тогда всюду, где $\omega \neq 0$, последнее уравнение равносильно следующему:

$$
(\mathbf{e} \cdot \boldsymbol{\nabla}) \mathbf{V}=\frac{1}{\omega}(\mathbf{V} \cdot \boldsymbol{\nabla}) \boldsymbol{\omega} .
$$

Рассмотрим произвольно выбранную вихревую линию, на которой скорость $\mathbf{V}=\mathbf{V}(l)$, где $l$ - переменная длина дуги вдоль этой вихревой линии. Тогда

$$
(\mathbf{e} \cdot \boldsymbol{\nabla}) \mathbf{V}=\frac{d}{d l} \mathbf{V}
$$

Обозначим через $V_{x}, V_{y}, V_{z}$ и $\omega_{x}, \omega_{y}, \omega_{z}$ компоненты вектора $\mathbf{V}$ и вектора завихренности $\boldsymbol{\omega}$ в некоторой прямоугольной декартовой системе координат Охуz. С использованием этих обозначений и равенства (5) векторное уравнение (4) на рассматриваемой вихревой линии можно записать в матричном виде

$$
\frac{d}{d l}\left(\begin{array}{c}
V_{x} \\
V_{y} \\
V_{z}
\end{array}\right)=\frac{1}{\omega}\left(\begin{array}{ccc}
\frac{\partial \omega_{x}}{\partial x} & \frac{\partial \omega_{x}}{\partial y} & \frac{\partial \omega_{x}}{\partial z} \\
\frac{\partial \omega_{y}}{\partial x} & \frac{\partial \omega_{y}}{\partial y} & \frac{\partial \omega_{y}}{\partial z} \\
\frac{\partial \omega_{z}}{\partial x} & \frac{\partial \omega_{z}}{\partial y} & \frac{\partial \omega_{z}}{\partial z}
\end{array}\right)\left(\begin{array}{c}
V_{x} \\
V_{y} \\
V_{z}
\end{array}\right)
$$

Коэффициенты матрицы зависят от вторых производных компонент скорости и являются непрерывными (и ограниченными) функциями на любом отрезке вихревой линии, где модуль завихренности $\omega \geqslant \omega_{0}>0$. Если считать

\footnotetext{
1 Замыкание нужно для того, чтобы рассматривать течение в том числе и на поверхности обтекаемого тела в окрестности точки торможения.
} 
коэффициенты матрицы заданными функциями переменной $l$, то матричное уравнение (6) представляет собой систему обыкновенных дифференциальных уравнений первого порядка для компонент вектора V. Эта система линейна, a ее коэффициенты непрерывны и ограничены. Поэтому из теоремы существования и единственности для систем обыкновенных дифференциальных уравнений [14] следует, что на всем рассматриваемом отрезке вихревой линии либо $|\mathbf{V}| \equiv 0$, либо $|\mathbf{V}| \neq 0$. Поскольку приведенные рассуждения верны для любого значения $\omega_{0}>0$, приходим к следующему выводу, который авторы предлагают назвать скоростной альтернативой.

Если на некотором отрезке вихревой линии стационарного движения идеальной несжимаемой жидкости завихренность не обращается в нуль, то величина скорости $|\mathbf{V}|$ на всем отрезке либо тождественно равна нулю, либо во всех точках отрезка $|\mathbf{V}| \neq 0$.

3. Скорость переноса линий тока. Дальнейшее исследование проведем с использованием критерия Зоравского [15]. В этом критерии говорится о переносе векторных линий частицами жидкости. Перенос векторных линий вектора с со скоростью а означает, что частицы жидкости, движущиеся со скоростью а и составляющие в некоторый момент времени векторную линию вектора с, продолжают составлять одну из векторных линий вектора с во все время своего движения. Согласно критерию Зоравского, для того чтобы векторные линии с переносились со скоростью а, необходимо и достаточно, чтобы во всем поле течения выполнялось равенство

$$
\mathbf{c} \times\left[\frac{\partial \mathbf{c}}{\partial t}+(\mathbf{a} \cdot \boldsymbol{\nabla}) \mathbf{c}-(\mathbf{c} \cdot \boldsymbol{\nabla}) \mathbf{a}\right]=0 .
$$

Чтобы использовать критерий Зоравского, рассмотрим в области $\bar{G}$ воображаемую жидкость, частицы которой движутся со скоростью $\boldsymbol{\omega}$. Из равенства (3) следует, что (7) выполнено для векторных линий вектора $\mathbf{V}$ и скорости воображаемой жидкости $\boldsymbol{\omega}$. Таким образом, если в некоторый момент времени частицы воображаемой жидкости, движущиеся со скоростью $\boldsymbol{\omega}$, составляли сегмент линии тока (то есть сегмент векторной линии V), содержащейся в области $\bar{G}$, то в любой последующий момент времени (пока эти частицы находятся в области $\bar{G}$ ) они будут составлять сегмент одной из линий тока жидкости. Другими словами, можно считать, что линии тока (реальной) жидкости движутся со скоростью $\boldsymbol{\omega}$.

4. Завихренность в точке торможения. Поскольку носовая часть гладкая и выпуклая, в некоторой окрестности точки торможения $C$ (см. рисунок) всюду, за исключением точки $C$, скорость $\mathbf{V}$ не равна нулю и все линии тока на поверхности тела (в этой окрестности) начинаются в точке $C$. В плоскопараллельных течениях точка торможения не единственная, и сформулированное утверждение не выполняется. Поэтому дальнейшее исследование проведем для неплоскопараллельных течений. ${ }^{2}$

Вдоль любой линии тока интеграл Бернулли $B$ сохраняет свое значение, поэтому на поверхности тела $B$ принимает одно и то же значение, равное своему значению в точке $C$. Следовательно, на поверхности тела (в рассматриваемой окрестности точки торможения) $\nabla B$ ортогонален этой поверхности.

\footnotetext{
${ }^{2}$ Плоскопараллельные течения будут обсуждены в следующем разделе.
} 
При этом во всех точках поверхности тела, кроме точки $C$, скорость $\mathbf{V}$ не равна нулю и лежит в касательной к этой поверхности плоскости. Поэтому из векторного уравнения (1) для всех точек поверхности тела, кроме точки $C$, следует, что завихренность $\boldsymbol{\omega}$ также лежит в касательной (к поверхности тела) плоскости. В силу непрерывности завихренность лежит в касательной плоскости и в точке $C$. Следовательно, в некоторой окрестности точки $C$ вихревые линии, имеющие хотя бы одну точку на поверхности тела, лежат на этой поверхности (если на этих вихревых линиях в окрестности точки $C$ завихренность не равна нулю).

Докажем, что завихренность $\boldsymbol{\omega}$ в точке торможения равна нулю. Предположим обратное, то есть, что в точке $C$ завихренность $\boldsymbol{\omega}$ не равна нулю. Тогда в силу непрерывности в некоторой окрестности точки $C$ завихренность $\boldsymbol{\omega}$ также не равна нулю. Значит, есть отрезок вихревой линии, лежащий на поверхности и проходящий через точку $C$, на котором скорость $\mathbf{V}=\mathbf{0}$ в точке $C$, а во всех других его точках $\mathbf{V} \neq \mathbf{0}$. Но это противоречит скоростной альтернативе (раздел 2). Полученное противоречие означает следующее.

При стационарном неплоскопараллельном обтекании идеальной несжимаемой жидкостью тела с гладкой выпуклой носовой частъю завихренность в точке торможения равна нулю.

5. О плоскопараллельных течениях. Как обычно, течение жидкости будем называть плоскопараллельным, если существует такая прямоугольная декартова система координат $O x y z$, в которой все гидродинамические параметры зависят только от координат $x$ и $y$, а $z$ - компонента скорости, всюду равная нулю. В таких течениях вихревые линии параллельны оси $z$ и на вихревых линиях гидродинамические параметры постоянны. Поэтому в плоскопараллельных течениях скоростная альтернатива (раздел 2) имеет место, но не дает ничего нового.

Использование того факта, что линии тока «движутся» со скоростью $\boldsymbol{\omega}$ (раздел 3), также приводит к известному результату. Действительно, частицы воображаемой жидкости, движущиеся со скоростью $\boldsymbol{\omega}$ и составляющие линию тока в какой-то момент времени, продолжают составлять линию тока. Все линии тока лежат в плоскостях вида $z=$ const. Следовательно, все частицы воображаемой жидкости, составляющие в какой-то момент времени линию тока, должны двигаться параллельно оси z с одинаковой скоростью $\boldsymbol{\omega}$. Это значит, что на каждой линии тока завихренность $\boldsymbol{\omega}$ постоянна, но это свойство давно известно, так как для плоскопараллельных течений оно легко следует из уравнения Гельмгольца [13].

Рассмотрим обтекание тела с гладкой выпуклой носовой частью вихревым потоком идеальной несжимаемой жидкости в случае плоскопараллельного течения. Как и в общем пространственном случае, поскольку носовая часть гладкая и выпуклая, на каждой плоскости вида $z=$ const скорость $\mathbf{V}$ равна нулю в единственной точке - точке торможения. Координаты $x$ и $y$ таких точек торможения одинаковы для всех плоскостей вида $z=$ const, поэтому точки торможения составляют линию, параллельную оси $z$. Если попытаться применить технику доказательства раздела 4, то предположение о ненулевой величине завихренности в точке торможения $C$ на одной из плоскостей вида $z=$ const не приведет к противоречию. Дело в том, что вихревая линия (параллельная оси $z$ ), проходящая через точку $C$, будет целиком лежать на теле и состоять из точек торможения, лежащих в других плоскостях вида 
$z=$ const, поэтому скорость на всей этой вихревой линии будет равна нулю, что не противоречит скоростной альтернативе (как это имело место в разделе 4).

Следовательно, вопрос о завихренности в точке торможения при обтекании тела с гладкой выпуклой носовой частью вихревым потоком идеальной несжимаемой жидкости в случае плоскопараллельного течения в данной работе не решен и остается открытым. В следующих разделах будем рассматривать неплоскопараллельные пространственные течения (в которых завихренность в точке торможения равна нулю).

6. Инвариант линии торможения. Рассмотрим движение частиц воображаемой жидкости, составляющих в некоторый момент времени $t_{0}$ линию торможения и двигающихся со скоростью $\boldsymbol{\omega}$. В точке торможения $\boldsymbol{\omega}$ равна нулю (раздел 4). Значит, частица воображаемой жидкости, находящаяся в этой точке, покоится. Предположим, что на линии торможения есть точки, в которых завихренность не равна нулю и имеет ненулевую нормальную к линии торможения составляющую. Тогда в последующий момент времени $t+\Delta t$ находившиеся в этих точках частицы воображаемой жидкости окажутся вне линии торможения и вместе с остальными частицами составят другую линию тока (раздел 3). Но тогда получается, что к точке торможения подходит еще одна (кроме линии торможения) линия тока, находящаяся во внутренних точках течения, что противоречит единственности линии торможения. Полученное противоречие показывает, что частицы воображаемой жидкости, составляющие линию торможения, либо покоятся, либо движутся вдоль этой линии. Следовательно, завихренность параллельна скорости, то есть вдоль всей линии торможения выполняется векторное равенство

$$
\boldsymbol{\omega} \times \mathbf{V}=0 .
$$

Рассмотрим скорость и завихренность на линии торможения. Скорость представим в виде

$$
\mathbf{V}=V(l) \mathbf{e}(l),
$$

где $|\mathbf{e}|=1, l$-переменная длина дуги вдоль линии торможения. На всей линии торможения, кроме точки $C$, скорость $V(l)$ не равна нулю. Поэтому в силу (8) завихренность можно представить в виде

$$
\boldsymbol{\omega}=\omega(l) \mathbf{e}(l)=\alpha(l) V(l) \mathbf{e}(l),
$$

где $\alpha$ - некоторая скалярная непрерывно дифференцируемая функция. Если направление возрастания длины дуги $l$ совпадает с направлением единичного вектора $\mathbf{e}$, то действие оператора $(\mathbf{e} \cdot \boldsymbol{\nabla})$ на линии торможения равносильно дифференцированию по $l$, т.е. $(\mathbf{e} \cdot \boldsymbol{\nabla}) \equiv \frac{d}{d l}$. Следовательно, на линии торможения (кроме точки $C$ ) уравнение (3) записывается в виде

$$
\alpha V \frac{d}{d l}(\mathbf{e} V)=V \frac{d}{d l}(\mathbf{e} \alpha V)
$$

или

$$
\alpha V^{2} \frac{d}{d l} \mathbf{e}+\alpha V \mathbf{e} \frac{d}{d l} V=\alpha V^{2} \frac{d}{d l} \mathbf{e}+\alpha V \mathbf{e} \frac{d}{d l} V+V^{2} \mathbf{e} \frac{d}{d l} \alpha
$$

После сокращений имеем $V^{2} \mathbf{e} \frac{d}{d l} \alpha=0$. Так как на всей линии торможения кроме точки торможения скорость не равна нулю, из последнего равенства 
получаем $\alpha=$ const, то есть $\omega / V=$ const. Таким образом, доказано следующее утверждение (инвариант линии торможения).

При стационарном неплоскопараллельном обтекании идеальной несжимаемой жидкостъю тела с гладкой выпуклой носовой частъю на всей линии торможения завихренность коллинеарна скорости, а отношение величинь завихренности $к$ величине скорости постоянно (кроме точки торможения, где это отношение не определено).

Заметим, что, согласно данной формулировке, инвариантом является и отношение $\omega / V$, и равное нулю векторное произведение $\boldsymbol{\omega} \times \mathbf{V}$.

7. О стационарном обтекании вихревым потоком. Рассмотрим стационарное обтекание вихревым потоком тела с гладкой выпуклой носовой частью (например шара), расположенного в канале, во всем входном сечении которого завихренность и скорость неколлинеарны. Тогда в том месте линии торможения, где она проходит через входное сечение, завихренность тоже должна быть неколлинеарна скорости. Однако из инварианта линии торможения, полученного в предыдущем разделе, следует, что завихренность и скорость коллинеарны вдоль всей линии торможения. Полученное противоречие вынуждает пересмотреть предположения, на которые опирается исследование, и заключить, что в данном случае обтекание не может быть стационарным. Рассмотренный пример показывает, что полученные в данной статье результаты могут применяться для качественного анализа течений.

Заключение. Исследована линия торможения в общем пространственном случае (за исключением плоскопараллельных течений) стационарного обтекания тела с гладкой выпуклой носовой частью несжимаемой идеальной жидкостью. Доказаны следующие три утверждения.

1. Если на некотором отрезке вихревой линии завихренность не обращается в нуль, то величина скорости жидкости на этом отрезке либо тождественно равна нулю, либо отлична от нуля во всех точках отрезка вихревой линии (скоростная альтернатива).

2. Завихренность в точке торможения равна нулю.

3. На линии торможения завихренность коллинеарна скорости и отношение величины завихренности к величине скорости одинаково во всех точках линии торможения (инвариант линии торможения).

Из третьего утверждения следует, что если в набегающем потоке скорость и завихренность неколлинеарны, то обтекание тела с гладкой выпуклой носовой частью обязательно будет нестационарным.

Полученные результаты могут быть использованы для качественного анализа течений и для верификации численных расчетов.

Конкурирующие интересы. Заявляем, что в отношении авторства и публикации этой статьи конфликта интересов не имеем.

Авторский вклад и ответственность. Все авторы принимали участие в разработке концепции статьи и в написании рукописи. Авторы несут полную ответственность за предоставление окончательной рукописи в печать. Окончательная версия рукописи была одобрена всеми авторами.

Финансирование. Исследование выполнялось без финансирования. 


\section{Библиографический список}

1. Aparinov A. A., Setukha A. V., Zhelannikov A. I. Numerical simulation of separated flow over three-dimensional complex shape bodies with some vortex method // AIP Conf. Proc., 2014. vol. 1629, 69. https://doi.org/10.1063/1.4902260.

2. Gutnikov V. A., Setukha A. V. Solving the problems of buildings and structures aerodynamics with a vortex method// IOP Conf. Ser.: Mater. Sci. Eng., 2018. vol. 456, 012068. https://doi.org/10.1088/1757-899X/456/1/012068.

3. Shcheglov G. A., Dergachev S. A. Vortex loops based method for subsonic aerodynamic loads calculation // MATEC Web Conf., 2018. vol.221, 05004. https://doi.org/10.1051/ matecconf /201822105004.

4. Il'ichev A. T., Tomashpolskii V. Ja. Characteristic parameters of nonlinear surface envelope waves beneath an ice cover under pre-stress // Wave Motion, 2019. vol. 86. pp. 11-20. https://doi.org/10.1016/j.wavemoti.2018.12.003.

5. Ильичев А. Т. Физические параметры уединенных волновых пакетов под ледовым покровом в бассейнах небольшой глубины // TMФ, 2019. Т. 201, № 3. С. 347-360. https:// doi.org/10.4213/tmf9746.

6. Marchenko A., Markov V., Taylor R. Influence of water on collisions of floating ice blocks: ISOPE-I-19-546: The 29th International Ocean and Polar Engineering Conference, 16-21 June, Honolulu, Hawaii, USA, 2019. 8 pp.

7. Сетуха А. В. О лагранжевом описании трехмерных течений вязкой жидкости при больших значениях числа Рейнольдса // Ж. вычисл. матем. и матем. физ., 2020. Т. 60, № 2 . C. 297-322. https://doi.org/10.31857/S004446692002012X.

8. Голубкин В. Н., Сизых Г. Б. Течение вязкого газа между вертикальными стенками // ПMM, 2018. T. 82, № 5. C. 657-667. https://doi.org/10.31857/S003282350002271-8.

9. Хорин А. Н., Конюхова А. А. Течение Куэтта горячего вязкого газа // Вестн. Сам. гос. техн. ун-та. Сер. Физ.-мат. науки, 2020. Т. 24, №2. С. 365-378. https://doi . org/10.14498/vsgtu1751.

10. Prosviryakov E. Yu. Exact solutions to generalized plane Beltrami-Trkal and Ballabh flows // Vestn. Samar. Gos. Tekhn. Univ., Ser. Fiz.-Mat. Nauki [J. Samara State Tech. Univ., Ser. Phys. Math. Sci.], 2020. vol. 24, no. 2. pp. 319-330. https://doi.org/10.14498/ vsgtu1766.

11. Kuzmina K., Marchevsky I., Ryatina E. Exact solutions of boundary integral equation arising in vortex methods for incompressible flow simulation around elliptical and Zhukovsky airfoils // J. Phys.: Conf. Ser., 2019. vol.1348, no. 1, 012099. https://doi.org/10.1088/ 1742-6596/1348/1/012099.

12. Сизых Г. Б. Значение энтропии на поверхности несимметричной выпуклой головной части при сверхзвуковом обтекании // ПММ, 2019. Т. 83, № 3. С. 377-383. https://doi . org/10.1134/S0032823519030135.

13. Лойцянский Л. Г. Механика жидкости и газа. М.: Дрофа, 2003. 840 с.

14. Понтрягин Л. С. Обыкновенные дифференииальные уравнения. Ижевск: Регулярная и хаотическая динамика, 2001. $400 \mathrm{c}$.

15. Prim R., Truesdell C. A derviation of Zorawski's criterion for permanent vector-lines // Proc. Amer. Math. Soc., 1950. vol.1, no.1. pp. 32-34. https://doi.org/10.2307/2032429. 


\title{
MSC: 76D17
}

\section{The invariant of stagnation streamline for a stationary vortex flow of an ideal incompressible fluid around a body}

\author{
(C) I. Yu. Mironyuk, L.A. Usov
}

\author{
Moscow Institute of Physics and Technology \\ (National Research University), \\ 9, Institutskiy per., Dolgoprudny, Moscow region, 141701, Russian Federation.
}

\begin{abstract}
In this study, using the Euler equations we investigate the stagnation streamline in the general spatial case of a stationary incompressible fluid flow around a body with a smooth convex bow. It is assumed that in some neighborhood of the stagnation point everywhere, except for the stagnation point, the fluid velocity is nonzero; and that all streamlines on the surface of the body in this neighborhood start at the stagnation point.

Here we prove the following three statements. 1) If on a certain segment of the vortex line the vorticity does not turn to zero, then the value of the fluid velocity in this segment is either identically equal to zero or nonzero at all points of the segment of the vortex line (velocity alternative). 2) The vorticity at the stagnation point is equal to zero. 3) On the stagnation streamline, the vorticity is collinear to the velocity, and the ratio of the vorticity to the velocity is the same at all points of the stagnation streamline (invariant of the stagnation streamline).

On the basis of the obtained results, it is concluded that if in the free stream the velocity and vorticity are not collinear, a stationary flow around the body is impossible. However, the question of vorticity at the stagnation point in plane-parallel flows remains open, because the accepted assumption that the velocity of the fluid differs from zero in some neighborhood of the stagnation point everywhere, except for the stagnation point itself, excludes plane-parallel flows from consideration.
\end{abstract}

Keywords: Euler equations, Helmholtz vortex theorems, Zorawski's criterion, stagnation streamline.

Received: $30^{\text {th }}$ July, $2020 /$ Revised: $15^{\text {th }}$ August, $2020 /$

Accepted: $16^{\text {th }}$ November, $2020 /$ First online: $25^{\text {th }}$ December, 2020

\section{Short Communication}

(ㅇ () (i) The content is published under the terms of the Creative Commons Attribution 4.0 International License (http://creativecommons.org/licenses/by/4.0/)

Please cite this paper in press as:

Mironyuk I. Yu., Usov L. A. The invariant of stagnation streamline for a stationary vortex flow of an ideal incompressible fluid around a body, Vestn. Samar. Gos. Tekhn. Univ., Ser. Fiz.-Mat. Nauki [J. Samara State Tech. Univ., Ser. Phys. Math. Sci.], 2020, vol. 24, no. 4, pp. 780-789. https://doi.org/10.14498/vsgtu1815 (In Russian).

\section{Authors' Details:}

Igor Yu. Mironyuk (1) https://orcid.org/0000-0002-3835-6059

Student; Faculty of Aeromechanics and Flight Engineering; e-mail:mironyuck.ig@yandex.ru

Lev A. Usov (1) https://orcid.org/0000-0001-7316-7328

Student; Faculty of Aeromechanics and Flight Engineering; e-mail: usov.la@phystech.edu 
Competing interests. We declare that we have no conflicts of interest in the authorship and publication of this article.

Authors' contributions and responsibilities. Each author has participated in the article concept development and in the manuscript writing. The authors are absolutely responsible for submitting the final manuscript in print. Each author has approved the final version of manuscript.

Funding. This research received no specific grant from any funding agency in the public, commercial, or not-for-profit sectors.

\section{References}

1. Aparinov A. A., Setukha A. V., Zhelannikov A. I. Numerical simulation of separated flow over three-dimensional complex shape bodies with some vortex method, AIP Conf. Proc., 2014, vol. 1629, 69. https://doi.org/10.1063/1.4902260.

2. Gutnikov V. A., Setukha A. V. Solving the problems of buildings and structures aerodynamics with a vortex method, IOP Conf. Ser.: Mater. Sci. Eng., 2018, vol. 456, 012068. https://doi.org/10.1088/1757-899X/456/1/012068.

3. Shcheglov G. A., Dergachev S. A. Vortex loops based method for subsonic aerodynamic loads calculation, MATEC Web Conf., 2018, vol.221, 05004. https://doi.org/10.1051/ matecconf /201822105004.

4. Il'ichev A. T., Tomashpolskii V. Ja. Characteristic parameters of nonlinear surface envelope waves beneath an ice cover under pre-stress, Wave Motion, 2019, vol. 86, pp. 11-20. https: // doi.org/10.1016/j. wavemoti.2018.12.003.

5. Il'ichev A. T. Physical parameters of solitary wave packets in shallow basins under ice cover, Theoret. and Math. Phys., 2019, vol.201, no.3, pp. 1710-1722. https://doi.org/ 10.1134/S0040577919120043.

6. Marchenko A., Markov V., Taylor R. Influence of water on collisions of floating ice blocks, ISOPE-I-19-546: The 29th International Ocean and Polar Engineering Conference, 16-21 June, Honolulu, Hawaii, USA, 2019, 8 pp.

7. Setukha A. V. Lagrangian description of three-dimensional viscous flowsat large Reynolds numbers, Comput. Math. and Math. Phys., 2020, vol.60, no. 2, pp. 302-326. https://doi. org/10.1134/S0965542520020116.

8. Golubkin V. N., Sizykh G. B. Viscous gas flow between vertical walls, Fluid. Dyn., 2018, vol. 53 (Suppl. 2), pp. 11-18. https://doi.org/10.1134/S0015462818060046.

9. Khorin A. N., Konyukhova A. A. Couette flow of hot viscous gas, Vestn. Samar. Gos. Tekhn. Univ., Ser. Fiz.-Mat. Nauki [J. Samara State Tech. Univ., Ser. Phys. Math. Sci.], 2020, vol. 24, no. 2, pp. 365-378 (In Russian). https://doi.org/10.14498/vsgtu1751.

10. Prosviryakov E. Yu. Exact solutions to generalized plane Beltrami-Trkal and Ballabh flows, Vestn. Samar. Gos. Tekhn. Univ., Ser. Fiz.-Mat. Nauki [J. Samara State Tech. Univ., Ser. Phys. Math. Sci.], 2020, vol. 24, no. 2, pp. 319-330. https://doi.org/10.14498/vsgtu1766.

11. Kuzmina K., Marchevsky I., Ryatina E. Exact solutions of boundary integral equation arising in vortex methods for incompressible flow simulation around elliptical and Zhukovsky airfoils, J. Phys.: Conf. Ser., 2019, vol.1348, no.1, 012099. https://doi.org/10.1088/ 1742-6596/1348/1/012099.

12. Sizykh G. B. Entropy value on the surface of a non-symmetric convex bow part of a body in the supersonic flow, Fluid. Dyn., 2019, vol.54, no.7, pp. 907-911. https://doi.org/ 10.1134/S0015462819070139.

13. Loitsyanskii L. G. Mechanics of Liquids and Gases. Oxford, Pergamon Press, 1966, 802 pp.

14. Pontryagin L. S. Obyknovennye differentsial'nye uravneniia [Ordinary Differential Equations]. Izhevsk, Regular and Chaotic Dynamics, 2001, 400 pp. (In Russian)

15. Prim R., Truesdell C. A derviation of Zorawski's criterion for permanent vector-lines, Proc. Amer. Math. Soc., 1950, vol. 1, no.1, pp. 32-34. https://doi.org/10.2307/2032429. 